\title{
Escalas de percepção da insegurança alimentar validadas: a experiência dos países da América Latina e Caribe
}

\author{
Perception scales of validated food insecurity: \\ the experience of the countries in Latin America and the Caribbean
}

Naiara Sperandio ${ }^{1}$

Dayane de Castro Morais ${ }^{2}$

Silvia Eloiza Priore ${ }^{2}$
${ }^{1}$ Universidade Federal do Rio de Janeiro. Av. Aluízio da Silva Gomes, Granja dos Cavaleiros. 27930560 Macaé RJ Brasil. naiarasperandio@ yahoo.com.br

${ }^{2}$ Departamento de Nutrição e Saúde, Universidade Federal de Viçosa. Viçosa MG Brasil.

\begin{abstract}
The scope of this systematic review was to compare the food insecurity scales validated and used in the countries in Latin America and the Caribbean, and analyze the methods used in validation studies. A search was conducted in the Lilacs, SciELO and Medline electronic databases. The publications were pre-selected by titles and abstracts, and subsequently by a full reading. Of the 16,325 studies reviewed, 14 were selected. Twelve validated scales were identified for the following countries: Venezuela, Brazil, Colombia, Bolivia, Ecuador, Costa Rica, Mexico, Haiti, the Dominican Republic, Argentina and Guatemala. Besides these, there is the Latin American and $\mathrm{Ca}$ ribbean scale, the scope of which is regional. The scales ranged from the standard reference used, number of questions and diagnosis of insecurity. The methods used by the studies for internal validation were calculation of Cronbach's alpha and the Rasch model; for external validation the authors calculated association and /or correlation with socioeconomic and food consumption variables. The successful experience of Latin America and the Caribbean in the development of national and regional scales can be an example for other countries that do not have this important indicator capable of measuring the phenomenon of food insecurity.
\end{abstract}

Key words Food and nutrition security, Validation studies, Latin America
Resumo Objetivou-se nesta revisão sistemática comparar as escalas de insegurança alimentar validadas e utilizadas nos países latino-americanos e caribenhos, e analisar os métodos empregados nos estudos de validação. Realizou-se busca nas bases eletrônicas Lilacs, SciELO e Medline. As publicações foram pré-selecionas pelos títulos e resumos, e posteriormente pela leitura integral. Dos 16.325 estudos revisados, selecionou-se 14. Foram identificadas 12 escalas validadas para os seguintes países: Venezuela, Brasil, Colômbia, Bolivia, Equador, Costa Rica, México, Haiti, República Dominicana, Argentina e Guatemala. Além dessas, tem-se a escala latino-americana e caribenha cuja abrangência é regional. As escalas variaram em relação ao padrão de referência utilizado, número de questões e diagnóstico da insegurança. Os métodos empregados pelos estudos para validação interna foi o cálculo do coeficiente alfa de Cronbach e o modelo Rasch; para validação externa os autores calcularam associação e/ou correlação com variáveis socioeconômicas e de consumo alimentar. A exitosa experiência da América Latina e Caribe no desenvolvimento de escalas nacionais e regionais pode ser exemplo para outros países que ainda não possuem esse importante indicador capaz de dimensionar o fenômeno da insegurança alimentar.

Palavras-chave Segurança alimentar e nutricional, Estudos de validação, América Latina 


\section{Introdução}

A segurança alimentar e nutricional (SAN) pode ser definida como o direito de todos ao acesso regular e permanente a alimentos de qualidade, em quantidade suficiente, sem comprometer o acesso a outras necessidades essenciais e que respeitem a diversidade cultural, sendo ambiental, cultural, econômica e socialmente sustentáveis ${ }^{1,2}$.

A abrangência desse conceito torna complexo sua mensuração e monitoramento, sendo necessária a utilização de diferentes indicadores, com comprovada validade e equivalência internacional, que contemplem as dimensões relacionadas a SAN, sendo elas, a da disponibilidade do alimento, do acesso físico e econômico, da utilização biológica do alimento/nutriente e da estabilidade que se refere ao elemento temporal das três dimensões citadas ${ }^{3,4}$.

Entre os indicadores comumente utilizados no meio científico de avaliação da SAN, destacam-se as escalas de percepção da insegurança alimentar, os de produção e consumo de alimentos, os antropométricos, socioeconômicos, bioquímicos e clínicos ${ }^{4}$. Esses indicadores devem, sempre que possível, serem utilizados de maneira complementar, uma vez que nenhum utilizado isoladamente será capaz de abarcar todas as dimensões que compõem o conceito de $\mathrm{SAN}^{3,4}$.

As escalas de percepção da insegurança alimentar, em relação aos outros indicadores de avaliação, sobressaem-se por possibilitar diagnóstico direto da situação de SAN, e classificação da gravidade da insegurança, que pode evoluir do nível mais leve (ausência da fome) até de maior gravidade (presença da fome) $)^{3,4}$.

Essas escalas são ferramentas importantes para mensuração da dimensão do acesso aos alimentos e vêm ganhando notoriedade internacional. Algumas características como ser de fácil aplicação, baixo custo, e basear-se na experiência vivenciada e percebida pelas famílias, reforçam sua utilização e aplicação em estudos de avaliação e monitoramento da insegurança alimentar ${ }^{5,6}$.

O Estados Unidos foi pioneiro no desenvolvimento de escala de percepção para avaliação da insegurança (experience - based food security scales $)^{5}$. Muitos países, em especial os da América Latina e Caribe, a partir da exitosa experiência americana, passaram a desenvolver estudos de validação, para dispor de instrumentos adequados que pudessem ser utilizados em suas populações. Essas experiências de validação no continente americano inspiraram iniciativas regionais, apoiadas pela Organização das Nações
Unidas para Alimentação e Agricultura (FAO), que culminaram na elaboração da Escala Latino -americana e Caribenha de Segurança Alimentar (ELCSA) ${ }^{6,7}$.

$\mathrm{Na}$ América Latina e Caribe muitos estudos fazem uso de escalas validadas de percepção da insegurança alimentar para avaliação e monitoramento desse fenômeno, sendo a única região do mundo a apresentar escala de abrangência regional (ELCSA) ${ }^{6,7,8}$. Isso reflete o compromisso da comunidade latino-americana e caribenha em identificar e monitorar a população em insegurança alimentar, o que pode servir como exemplo para outras regiões do mundo ${ }^{7,8}$.

Diante disso, o objetivo desta revisão sistemática foi comparar as escalas de percepção da insegurança alimentar validadas e utilizadas nos países da América Latina e Caribe, assim como analisar os métodos empregados nos estudos de validação das mesmas.

\section{Metodologia}

A pergunta norteadora para realização desta revisão sistemática foi: "Quais países da América Latina e Caribe possuem escalas de percepção para diagnóstico da insegurança alimentar validadas, e quais foram os métodos empregados nos estudos de validação das mesmas?"

\section{Critérios de inclusão e exclusão dos artigos}

Foram analisados estudos originais, realizados na América Latina e Caribe, e indexados nas bases de dados: SciELO, Science Direct e PubMed (Lilacs e Medline), além de materiais oficiais referentes ao processo de validação e utilização dessas escalas em seus respectivos países. Consultou-se também as listas de referências dos artigos (busca reversa), a fim de localizar outros estudos publicados e não identificados na busca inicial.

Incluiu-se estudos que utilizavam escalas de percepção para diagnóstico da insegurança alimentar e que explicitavam o processo de validação dessas escalas nos países latino-americanos e caribenhos. Não se limitou o período e o idioma de publicação. Os estudos que não atenderam aos critérios de inclusão foram excluídos, assim como artigos de revisão, dissertações ou teses.

\section{Busca e seleção dos artigos}

Os termos indexadores utilizados, de forma combinada, foram: segurança alimentar, segu- 
rança alimentar e nutricional, estudos de validação, escala e percepção, assim como seus respectivos vocábulos em inglês (food security, food and nutrition security, validation studies, scale e perception) e em espanhol (seguridad alimentaria, seguridad alimentaria y nutricional, estudios de validación, escala e percepción).

A busca eletrônica em base de dados identificou incialmente 16.325 artigos. A partir da leitura do título realizou-se o primeiro refinamento da pesquisa, onde excluiu-se 16.259 estudos que não abordavam o tema dessa revisão (trabalhos que utilizavam escalas não validadas, ou que propunham novos instrumentos de avaliação da insegurança alimentar, artigos de revisão, e estudos repetidos) (Figura 1).

Selecionou-se 66 estudos para leitura do resumo, e outros 4 identificados na lista de referências dos mesmos. A partir da leitura dos resumos, realizou-se o segundo refinamento, excluindo
50 do total. Incluiu-se 20 artigos na última etapa de seleção, que foram analisados na íntegra. O último refinamento excluiu 6 estudos, devido, principalmente, aos procedimentos metodológicos empregados, que não possibilitavam compreender o processo de validação das escalas de percepção.

A partir das três etapas de refinamento, utilizou-se, para realização desta revisão sistemática, 14 estudos, sendo que desses, 9 são artigos originais e 5 referem-se a materiais científicos sobre as escalas da República Dominicana, Argentina, México, Guatemala e ELCSA.

Os trabalhos foram agrupados e analisados de acordo com o país e ano de validação da escala, número de questões do instrumento, critérios de classificação propostos para diagnóstico da insegurança alimentar, assim como os padrões de referência e métodos utilizados para validação dos instrumentos.

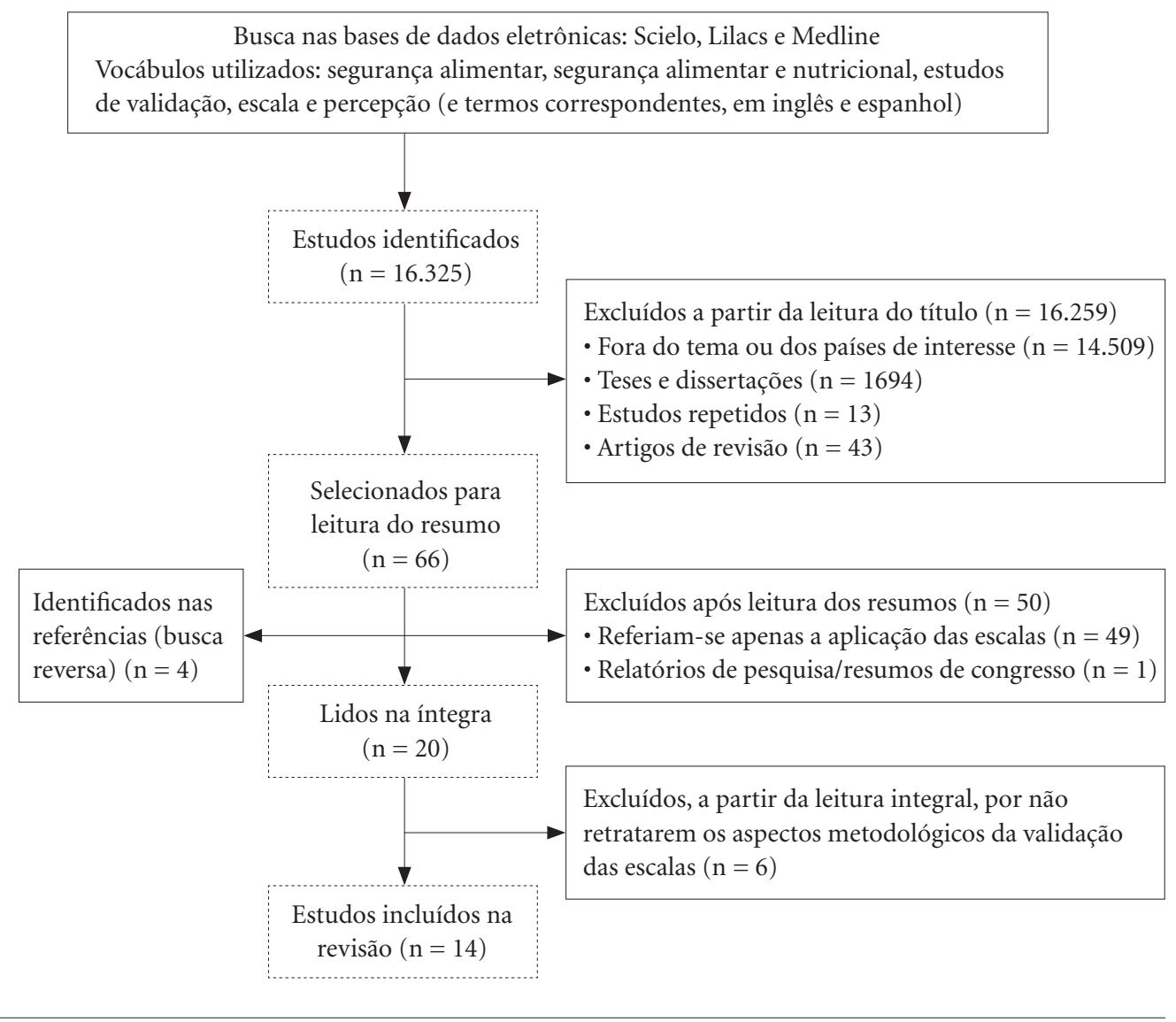

Figura 1. Protocolo de elaboração da revisão sistemática. 


\section{Resultados}

Foram encontradas, nesta revisão: 12 escalas de insegurança alimentar validadas. Os países que apresentavam essas escalas foram Venezuela $^{9}$, Brasil (nas versões longa e reduzida) ${ }^{10,11}$, Colômbia $^{12}$, Bolívia ${ }^{13}$, Equador ${ }^{14}$, Costa Rica ${ }^{15}$, México $^{16}$, Haiti ${ }^{17}$, República Dominicana ${ }^{18}$, Argentina $^{19}$ e Guatemala ${ }^{20}$. Ressalta-se, além das escalas específicas por país, a América Latina e o Caribe possuem escala própria, denominada Escala Latino-americana e Caribenha de Segurança Alimentar $^{21,22}$ (Quadro 1).

Os estudos de validação dessas escalas, nos países latino-americanos e caribenhos, surgiram a partir da década de 2000. A “Escala de Seguridad Alimentaria Percibida” foi o primeiro instrumento validado, em 2000, na Venezuela ${ }^{9}$, sendo utilizada com as mesmas características na Colômbia, a partir de $2006^{12}$.

Em relação ao número de questões presentes nas escalas, observou-se variação entre 5 a 16 itens. Escalas com 5 perguntas são utilizadas na Argentina $^{19}$ e no Brasil ${ }^{11}$, sendo que nesse último caso, trata-se da versão reduzida da Escala Brasileira de Insegurança Alimentar (EBIA). As escalas com 16 itens são usadas no Haiti ${ }^{17}$ e República Dominicana ${ }^{18}$.

A maioria dos instrumentos possui perguntas adicionais específicas, quando há no domicílio presença de crianças ou jovens, exceto a da Bolívia $^{13}$ e a versão curta da EBIA, no Brasil ${ }^{20}$. O tempo de referência também variou entre os instrumentos (30 dias; 3, 6 e 12 meses). Em relação a pontuação, na sua maioria, respostas afirmativas as questões contabilizam um ponto, e os critérios de diagnóstico possibilitam determinar os níveis de gravidade da insegurança (leve, moderada e grave; insegurança alimentar sem fome e insegurança alimentar com fome). As escalas da Venezuela ${ }^{9}$, Colômbia $^{12}$, Bolívia ${ }^{13}$, Equador ${ }^{14}$ e Argentina ${ }^{19}$ abordam também a frequência de ocorrência do evento em questão (sempre/muitas vezes; algumas vezes/as vezes; raramente).

Em relação às características dos estudos de validação das escalas de percepção da insegurança alimentar (Quadro 2), a maioria utilizou como padrão de referência instrumentos desenvolvidos e utilizados nos Estados Unidos, para avaliação desse fenômeno, sendo eles Community Childhood Hunger Identification Project (CCHIP) ${ }^{9}$ e Household Food Security Supl.emental Module $(\text { HFSSM })^{10,13,14,15,18}$. México ${ }^{16}$, Haiti $^{17}$, Argentina ${ }^{19}$ e Guatemala $^{20}$ utilizaram a ELCSA como padrão de referência para o desenvolvimento de suas escalas.
Para elaboração da ELCSA foram utilizadas como padrão de referência as escalas da Venezuela $^{9}$, Brasil $^{10}$ e Colômbia ${ }^{12}$. Os primeiros estudos que utilizaram essa escala para confirmar sua validade foram realizados por Álvarez et al. ${ }^{21}$ (2008), na Colômbia, e Pérez-Escamilla et al. ${ }^{22}$ (2011), no México e Uruguai.

O tamanho amostral dos estudos variou de 52 domicílios, no Equador ${ }^{14}$, até 265.212, na Guatemala $^{20}$. O processo de validação interna envolveu, especialmente, o modelo matemático Rasch $^{12,14,16,17,18,20}$ e o cálculo do Coeficiente Alfa de Cronbach $9,10,21,22,15,17$, sendo que os valores apresentados foram satisfatórios para ambos os testes (valores de infit entre 0,8 a 1,2 no modelo Rasch e coeficiente alfa de Cronbach maior que 0,85).

Em alguns países, como na Bolívia ${ }^{13}$ e Argentina ${ }^{19}$ os estudos não apresentaram metodologia e resultados acerca da validação interna das escalas. Para testar a validade da versão curta da EBIA, no Brasil, os autores optaram por calcular sensibilidade, especificidade e coeficiente de Kap$\mathrm{pa}^{11}$.

Em relação a validação externa, que analisa a validade preditiva das escalas, os métodos empregados foram, principalmente, análises de correlação e/ou associação com variáveis socioeconômicas, enfatizando aquelas relacionadas a pobreza, como, por exemplo, renda familiar, e indicadores do consumo alimentar.

A abrangência dos estudos, em determinados países, envolveu apenas domicílios de áreas urbanas, como foi o caso da Venezuela ${ }^{9}$, Costa Rica ${ }^{15}$ e Argentina ${ }^{19}$, ou apenas domicílios rurais, que foi o caso do Equador ${ }^{14}$, Haiti ${ }^{17}$ e República Dominicana $^{18}$. Nos demais estudos a abrangência envolveu domicílios urbanos e rurais.

\section{Discussão}

A utilização de escalas psicométricas como indicador direto de avaliação e monitoramento da insegurança alimentar representa importante avanço para compreensão desse fenômeno multidimensional e a possibilidade de determinação de grupos populacionais vulneráveis a violação do direito humano à alimentação adequada e saudável (DHAA) ${ }^{23}$.

Indicadores indiretos, especialmente os relacionados a disponibilidade de alimentos, vêm sendo historicamente utilizados para avaliação da insegurança. No entanto, esses indicadores, de maneira geral, refletem mais os determinantes da 
Quadro 1. Características das escalas de percepção de insegurança alimentar utilizadas nos países latino-americanos e caribenhos.

\begin{tabular}{|c|c|c|c|}
\hline $\begin{array}{c}\text { Escala e ano } \\
\text { de elaboração }\end{array}$ & $\begin{array}{l}\text { Países que } \\
\text { utilizam }\end{array}$ & Número de questões & $\begin{array}{c}\text { Classificação, segundo pontuação } \\
\text { (Respostas afirmativas as perguntas) }\end{array}$ \\
\hline $\begin{array}{l}\text { Escala de } \\
\text { Seguridad } \\
\text { Alimentaria } \\
\text { Percibida, } 2000^{9}\end{array}$ & Venezuela & $\begin{array}{l}\text { - } 12 \text { questões, quando presença de } \\
\text { menor de } 10 \text { anos } \\
\text { - } 7 \text { questões, quando não presença de } \\
\text { menor de } 10 \text { anos } \\
\text { - Para cada item a pessoa responde } \\
\text { sim ou não. Quando há resposta } \\
\text { afirmativa, pergunta-se se isto ocorre } \\
\text { sempre ( } 3 \text { pontos), algumas vezes } \\
\text { (2 pontos), ou raramente (1 ponto), } \\
\text { somando máximo de } 36 \text { pontos. }\end{array}$ & $\begin{array}{l}\text { - Segurança alimentar = 0; Insegurança } \\
\text { alimentar leve = 1-12; Insegurança alimentar } \\
\text { moderada =13-24; Insegurança alimentar } \\
\text { grave = mais do que } 25 \text { pontos. } \\
\text { - Segurança alimentar = } 0 \text {; Insegurança } \\
\text { alimentar leve = 1-7; Insegurança alimentar } \\
\text { moderada = 8-14; Insegurança alimentar } \\
\text { grave = mais do que } 15 \text { pontos. } \\
\text { *Tempo de referência: } 6 \text { últimos meses }\end{array}$ \\
\hline $\begin{array}{l}\text { Escala Brasileira } \\
\text { de Insegurança } \\
\text { Alimentar (EBIA), } \\
2004^{10} \\
\text { - versão longa }\end{array}$ & Brasil & $\begin{array}{l}\text { - } 15 \text { questões, quando presença de } \\
\text { menor de } 18 \text { anos } \\
\text { - } 8 \text { questão, quando não presença de } \\
\text { menor de } 18 \text { anos } \\
\text { *Respostas: sim ou não, sendo que } \\
\text { cada resposta afirmativa corresponde } \\
\text { a } 1 \text { ponto }\end{array}$ & $\begin{array}{l}\text { - Segurança alimentar }=0 \text {; Insegurança } \\
\text { alimentar leve }=1-5 ; \text { Insegurança alimentar } \\
\text { moderada }=6-10 \text {; Insegurança alimentar } \\
\text { grave }=11-15 . \\
\text { - Segurança alimentar }=0 \text {; Insegurança } \\
\text { alimentar leve = 1-3; Insegurança alimentar } \\
\text { moderada }=4-6 ; \text { Insegurança alimentar } \\
\text { grave }=7-8 . \\
\text { *Tempo de referência: } 3 \text { últimos meses } \\
\end{array}$ \\
\hline $\begin{array}{l}\text { Escala de } \\
\text { Percepción } \\
\text { de Seguridad } \\
\text { Alimentaria } \\
\text { (EPSA), } 2006^{12} \\
\end{array}$ & Colômbia & Idem Venezuela & Idem Venezuela \\
\hline $\begin{array}{l}\text { U.S. Household } \\
\text { Food Security } \\
\text { Supplemental } \\
\text { Module (HFSSM) } \\
\text { version, } 2006^{13}\end{array}$ & Bolívia & $\begin{array}{l}\text { - } 9 \text { questões } \\
\text { - Para cada item a pessoa responde } \\
\text { sim ou não. Quando há resposta } \\
\text { afirmativa, pergunta-se a frequência } \\
\text { de ocorrência (muitas vezes, às } \\
\text { vezes, ou raramente). Para respostas } \\
\text { afirmativas e frequência de ocorrência } \\
\text { "muitas vezes" ou “às vezes", pontua- } \\
\text { se } 1 \text {; para as respostas negativas, ou } \\
\text { para frequência "raramente" pontua- } \\
\text { se } 0 \text {. }\end{array}$ & $\begin{array}{l}\text { - Segurança alimentar }=0 \text {; Insegurança } \\
\text { alimentar leve }=1-2 ; \text { Insegurança alimentar } \\
\text { moderada }=3-5 ; \text { Insegurança alimentar } \\
\text { grave }=6-9 . \\
\text { *Tempo de referência: últimos } 12 \text { meses }\end{array}$ \\
\hline $\begin{array}{l}\text { Adult and Children } \\
\text { Food Security } \\
\text { Survey, } 2007^{14}\end{array}$ & Equador & $\begin{array}{l}\text { - } 8 \text { questões, quando presença de } \\
\text { menor de } 18 \text { anos } \\
\text { - } 7 \text { questões, quando não presença de } \\
\text { menor de } 18 \text { anos } \\
\text { *Respostas: sim ou não, sendo que } \\
\text { cada resposta afirmativa corresponde } \\
\text { a } 1 \text { ponto. }\end{array}$ & $\begin{array}{l}\text { - Segurança alimentar }=0 \text {; Insegurança } \\
\text { alimentar sem fome }=1-3 \text {; Insegurança com } \\
\text { fome }=4-8 \text {. } \\
\text { - Segurança alimentar }=0 \text {; Insegurança } \\
\text { alimentar com fome }=1-3 \text {; Insegurança sem } \\
\text { fome }=4-7 \text {. } \\
\text { ^Tempo de referência: últimos } 12 \text { meses }\end{array}$ \\
\hline
\end{tabular}

continua

insegurança alimentar e suas consequências para saúde, do que de fato as dificuldades vivenciadas pelas famílias em relação ao acesso a alimentação adequada e saudávelel 23,24 . 


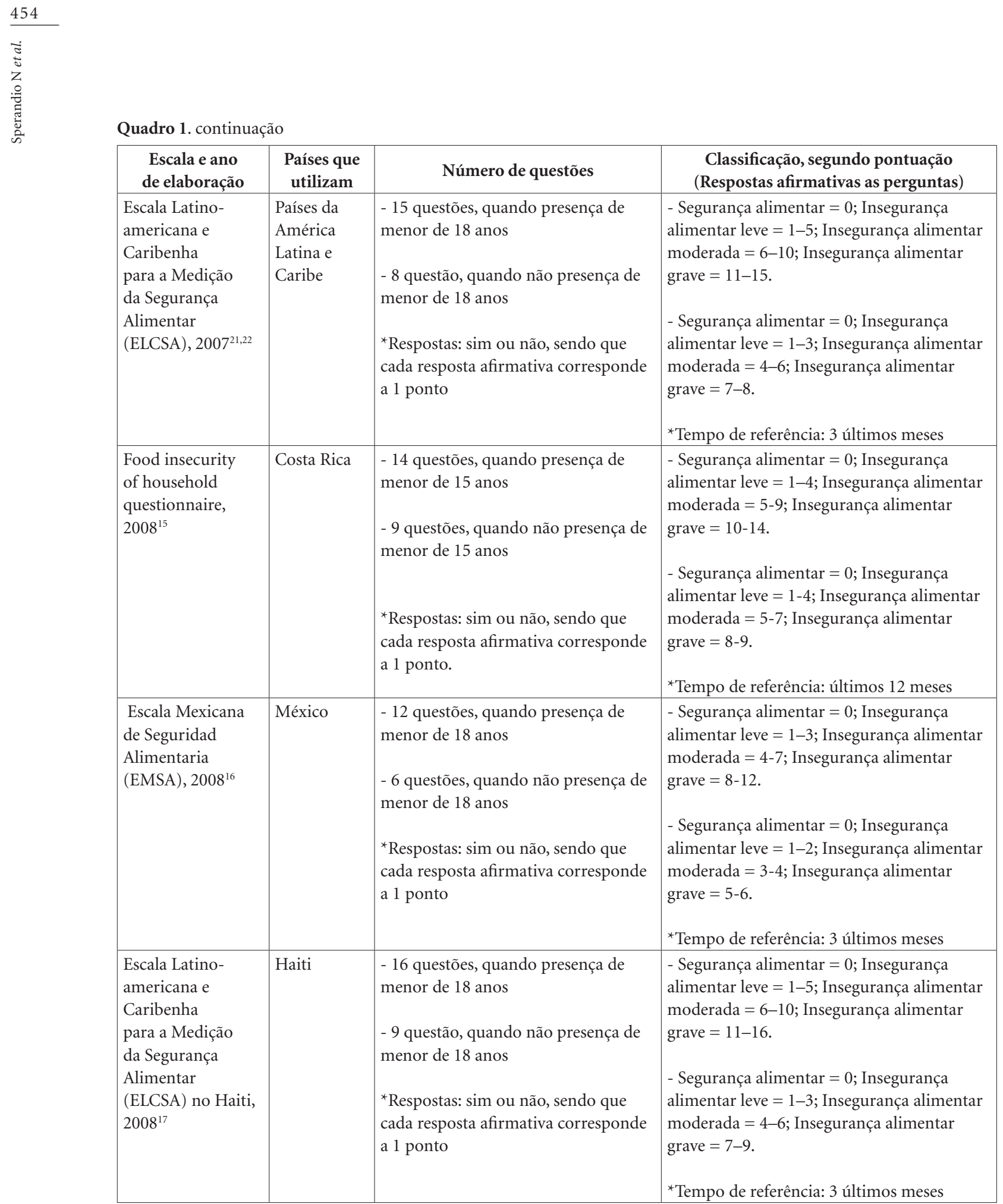

continua

Os primeiros estudos de validação de escalas de percepção da insegurança alimentar na América Latina e Caribe utilizaram como padrão de referência escalas desenvolvidas no Estados Unidos (EUA), que foi pioneiro no desenvolvimento desses instrumentos ${ }^{5,25}$.
A partir do estudo de Radimer/Cornell ${ }^{5}$ e a da Community Childhood Hunger Identification Project - CCHIP ${ }^{26}$, pesquisadores reunidos pelo United States Department of Agriculture (USDA) desenvolveram uma escala válida para aplicação em âmbito nacional, denominada Household 
Quadro 1. continuação

\begin{tabular}{|c|c|c|c|}
\hline $\begin{array}{c}\text { Escala e ano } \\
\text { de elaboração }\end{array}$ & $\begin{array}{l}\text { Países que } \\
\text { utilizam }\end{array}$ & Número de questões & $\begin{array}{c}\text { Classificação, segundo pontuação } \\
\text { (Respostas afirmativas as perguntas) }\end{array}$ \\
\hline $\begin{array}{l}\text { Dominican } \\
\text { Republic } \\
\text { Household Food } \\
\text { Security Survey } \\
\text { Module, } 2008^{18}\end{array}$ & $\begin{array}{l}\text { República } \\
\text { Dominicana }\end{array}$ & $\begin{array}{l}\text { - } 16 \text { questões, quando presença de } \\
\text { menor de } 18 \text { anos } \\
\text { - } 8 \text { questões, quando não presença de } \\
\text { menor de } 18 \text { anos } \\
\text { ^Respostas: Até a sétima pergunta, } \\
\text { existem as opções de resposta: } \\
\text { "frequentemente verdade", "algumas } \\
\text { vezes verdade" e "nunca é verdade"; a } \\
\text { partir da oitava pergunta, as opções } \\
\text { de resposta são sim ou não. As opções } \\
\text { de resposta "frequentemente verdade" } \\
\text { e "algumas vezes verdade" pontuam } \\
\text { como respostas afirmativas. }\end{array}$ & $\begin{array}{l}\text { - Segurança alimentar }=0 \text {; Insegurança sem } \\
\text { fome }=1-2 ; \text { Insegurança com fome }=3-16 . \\
\text { - Segurança alimentar }=0 \text {; Insegurança sem } \\
\text { fome }=1-2 ; \text { Insegurança com fome }=3-8 . \\
\text { * Tempo de referência: últimos } 30 \text { dias }\end{array}$ \\
\hline $\begin{array}{l}\text { Del Módulo } \\
\text { de Inseguridad } \\
\text { Alimentaria } \\
\text { Encuesta } \\
\text { de la Deuda } \\
\text { Social (EDSA) } \\
\text { Bicentenario, } \\
2010^{19}\end{array}$ & Argentina & $\begin{array}{l}\text { - } 5 \text { questões, quando presença de } \\
\text { menor de } 17 \text { anos } \\
\text { - } 3 \text { questões, quando não presença de } \\
\text { menor de } 17 \text { anos } \\
\text { *Respostas: sim ou não nas questões } \\
1 \text { a } 4 \text {, sendo que respostas afirmativas } \\
\text { correspondem a 1, } 2,3 \text { e } 4 \text { pontos, } \\
\text { respectivamente; e na questão } 5 \text { as } \\
\text { respostas "muitas vezes" e "várias } \\
\text { vezes" equivalem a } 2 \text { pontos, "alguma } \\
\text { vez" a } 1 \text { ponto e "nunca" a } 0 \text { ponto, }\end{array}$ & $\begin{array}{l}\text { - Segurança alimentar }=0 \text {-3; Insegurança } \\
\text { alimentar moderada }=4-7 \text {; Insegurança } \\
\text { alimentar grave }=8-12 \text {. } \\
\text { - Segurança alimentar }=0 \text {-2; Insegurança } \\
\text { alimentar moderada }=3 \text {-4; Insegurança } \\
\text { alimentar grave }=5 . \\
\text { ^Tempo de referência: } 12 \text { últimos meses }\end{array}$ \\
\hline $\begin{array}{l}\text { Escala Latino- } \\
\text { americana e } \\
\text { Caribenha } \\
\text { para a Medição } \\
\text { da Segurança } \\
\text { Alimentar } \\
\text { (ELCSA) na } \\
\text { Guatemala, } 2010^{20}\end{array}$ & Guatemala & $\begin{array}{l}\text { - } 15 \text { questões, quando presença de } \\
\text { menor de } 18 \text { anos } \\
\text { - } 8 \text { questão, quando não presença de } \\
\text { menor de } 18 \text { anos } \\
\text { ^Respostas: sim ou não, sendo que } \\
\text { cada resposta afirmativa corresponde } \\
\text { a } 1 \text { ponto }\end{array}$ & $\begin{array}{l}\text { - Segurança alimentar }=0 \text {; Insegurança } \\
\text { alimentar leve }=1-5 \text {; Insegurança alimentar } \\
\text { moderada }=6-10 \text {; Insegurança alimentar } \\
\text { grave }=11-15 \text {. } \\
\text { - Segurança alimentar }=0 \text {; Insegurança } \\
\text { alimentar leve = 1-3; Insegurança alimentar } \\
\text { moderada }=4-6 \text {; Insegurança alimentar } \\
\text { grave }=7-8 . \\
\star \text { Tempo de referência: } 3 \text { últimos meses } \\
\end{array}$ \\
\hline $\begin{array}{l}\text { Escala Brasileira } \\
\text { de Insegurança } \\
\text { Alimentar (EBIA), } \\
2014^{11} \\
\text {-versão curta }\end{array}$ & Brasil & $\begin{array}{l}-1^{\text {a }} \text { proposta: } 7 \text { questões, } \\
\text { independente da presença de menor } \\
\text { de } 18 \text { anos } \\
-2^{\text {a }} \text { proposta: } 5 \text { questões, } \\
\text { independente da presença de menor } \\
\text { de } 18 \text { anos } \\
\text { ^Respostas: sim ou não, sendo que } \\
\text { cada resposta afirmativa corresponde } \\
\text { a } 1 \text { ponto }\end{array}$ & $\begin{array}{l}\text { - Escala de } 7 \text { questões: Segurança } \\
\text { alimentar=0; Insegurança alimentar }=1-7 \text {. } \\
\text { - Escala de } 5 \text { questões: Segurança } \\
\text { alimentar=0; Insegurança alimentar }=1-5 \text {. } \\
\text { ^Tempo de referência: } 3 \text { últimos meses }\end{array}$ \\
\hline
\end{tabular}


Food Security Survey Module - HFSSM, composta por 18 itens. A partir de 1995, esse instrumento passou a ser utilizado em pesquisas americanas, como por exemplo, a National Health and Nutrition Examination Survey (NHANES) ${ }^{25}$.

A extensão da HFSSM limitava sua utilização em pesquisas americanas, principalmente aquelas com restrições financeiras e de menor duração. Em decorrência disso, Blumber et al. ${ }^{27}$ desenvolveram uma versão curta de seis itens, que apresentou boa concordância com a escala longa, podendo ser usada como screening na avaliação da insegurança alimentar.

Semelhante aos EUA, o Brasil também possui versão curta da EBIA, publicada em $2014^{11}$, tornando-o pioneiro em relação aos outros países latino-americanos e caribenhos. Além da nova proposta de versão curta, a EBIA sofreu modificação em 2009, com exclusão de uma questão que se referia a perda de peso entre os moradores do domicílio, logo, a EBIA utilizada atualmente no Brasil, contêm 14 questões $^{28}$. Isso aconteceu em decorrência do processo de transição nutricional vivenciado pelo país, onde a prevalência de excesso de peso vem se tornando cada vez maior, inclusive entre as famílias de baixa renda ${ }^{29}$.

Os estudos de validação apresentados, de maneira geral, utilizaram de metodologias que foram além da simples tradução do padrão de referência para o idioma do país ao qual se pretendia desenvolver a escala. Além do processo de tradução, os estudos realizaram validação interna do instrumento, analisada pelo coeficiente alfa de Cronbach e Modelo Rasch, ambos recomendados para esse tipo de análise ${ }^{30,31}$; e validação externa que envolveu a aplicação da escala em inquéritos populacionais e o uso de métodos de associação e correlação com variáveis socioeconômicas e de consumo alimentar.

Alguns estudos não apresentaram resultados de validação interna das escalas, como foi o caso da Bolívia e Argentina. Em relação a validação da versão curta da EBIA no Brasil, os autores optaram pela utilização de testes de diagnóstico (avaliação da sensibilidade e especificidade do instrumento) e testes de concordância (coeficiente de Kappa).

O processo de validação interna de um instrumento, como no caso de escalas psicométricas, é essencial para garantir confiabilidade e adequabilidade do mesmo. O coeficiente alfa de Cronbach permite avaliar a consistência interna das respostas aos itens da escala e indicar se as mesmas estavam correlacionadas entre si, sendo o valor mínimo aceitável de $0,7^{30}$. Conforme os resultados do Quadro 2, todos os estudos que avaliaram validade pelo alfa de Cronbach, apresentaram boa consistência interna das escalas.

O modelo Rasch, dentre outros objetivos, pode ser utilizado para verificação da unidimensionalidade do instrumento, isto é, se todos os itens presentes estão mensurando um único constructo ou fator, que no caso seria a insegurança alimentar. No modelo, espera-se que famílias que não vivenciam a insegurança tenham menor probabilidade de responder positivamente aos itens, enquanto que, aquelas que vivenciam respondam mais positivamente. Como as escalas avaliadas mensuravam apenas um constructo (insegurança alimentar), elas foram analisadas segundo os valores de ajuste (Infit), sendo que todas que utilizaram este modelo para validação apresentaram valores adequados $(0,8 \text { a } 1,2)^{31}$.

A FAO recomenda que nos estudos de validação de escalas psicométricas sejam calculados o coeficiente de alfa de Cronbach e o modelo de Rasch para garantir maior confiabilidade dos novos instrumentos propostos ${ }^{32}$. A maioria dos estudos de validação das escalas utilizadas nos países latino-americanos e caribenhos, analisados nesta revisão, seguiram essa recomendação da FAO.

Ressalta-se que no Brasil ${ }^{10,11}$, Colômbia ${ }^{12}$, Bolívia $^{13}$, México ${ }^{16}$ e Guatemala ${ }^{20}$, o processo de validação envolveu domicílios da zona urbana e rural dos respectivos países. A participação na amostra de domicílios urbanos e rurais é importante, uma vez que, os mesmos representam distintos contextos culturais, sociais e econômicos que podem influenciar a ocorrência da insegurança alimentar. Além disso, o país poderá dispor de um instrumento único que poderá ser aplicado independentemente da situação dos domicílios (urbano ou rural).

A exitosa experiência no processo de validação das escalas de insegurança alimentar, especialmente na Venezuela, Colômbia e Brasil, foram referência para elaboração da ELCSA, que contou com o apoio da $\mathrm{FAO}^{21,22,32}$. A ELCSA representa importante ferramenta para compreensão da distribuição, causas e consequências da insegurança alimentar na América Latina e Caribe. Desde sua publicação em 2007, a ELCSA vem sendo utilizada em estudos sobre segurança alimentar, pesquisas nacionais e em avaliações de políticas e programas sociais ${ }^{32}$. Análises recentes confirmam a confiabilidade e a validade dessa escala, que somado ao seu baixo custo, a fácil aplicação e uniformidade em toda América Latina e Caribe, a tornou importante indicador regional ${ }^{21,22}$. 
Quadro 2. Características dos estudos que validaram as escalas de percepção em países latino-americanos e caribenhos.

\begin{tabular}{|c|c|c|c|c|c|}
\hline Referência & Escala & País, ano & $\begin{array}{l}\text { Padrão de } \\
\text { referência para } \\
\text { validação } \\
\end{array}$ & $\begin{array}{l}\text { Método de validação e } \\
\text { amostra utilizada }\end{array}$ & Principais resultados \\
\hline $\begin{array}{l}\text { Dellohain e } \\
\text { Sanjur }^{9}\end{array}$ & $\begin{array}{l}\text { Escala de } \\
\text { Seguridad } \\
\text { Alimentaria } \\
\text { Percibida }\end{array}$ & $\begin{array}{l}\text { Venezuela, } \\
2000\end{array}$ & $\begin{array}{l}\text { Community } \\
\text { Childhood Hunger } \\
\text { Identification } \\
\text { Project (CCHIP - } \\
\text { EUA) }\end{array}$ & $\begin{array}{l}\text { - Calculou-se o alfa } \\
\text { de Cronbach para } \\
\text { validação interna; } \\
\text { realizou-se análise de } \\
\text { regressão múltipla } \\
\text { relacionando possíveis } \\
\text { determinantes, } \\
\text { econômicos, sociais e } \\
\text { comportamentais com } \\
\text { o nível de insegurança } \\
\text { alimentar medido pela } \\
\text { escala } \\
\text { - Primeira etapa do } \\
\text { processo de validação } \\
\text { foi um estudo piloto } \\
\text { para testar a adaptação } \\
\text { e a confiabilidade da } \\
\text { nova escala com } 65 \\
\text { mulheres. A segunda } \\
\text { etapa foi um estudo de } \\
\text { campo envolvendo } 238 \\
\text { famílias }\end{array}$ & $\begin{array}{l}\text { - Valor do alfa de } \\
\text { Cronbach foi de } 0,87 ; \\
\text { variáveis preditoras, } \\
\text { suficiência energética, } \\
\text { renda mensal per capita, } \\
\text { classe social e número } \\
\text { de filhos no domicílio, } \\
\text { associaram-se, em direção } \\
\text { esperada, com a escala de } \\
\text { insegurança }\left(\mathrm{R}^{2}=0,343\right)\end{array}$ \\
\hline $\begin{array}{l}\text { Pérez- } \\
\text { Escamilla et } \\
\text { al. }^{10}\end{array}$ & $\begin{array}{l}\text { Escala Brasileira } \\
\text { de Insegurança } \\
\text { Alimentar (EBIA) }\end{array}$ & Brasil, 2004 & $\begin{array}{l}\text { U.S. Household } \\
\text { Food Security } \\
\text { Supplemental } \\
\text { Module (HFSSM) }\end{array}$ & $\begin{array}{l}\text { - Realização de grupos } \\
\text { focais para avaliação } \\
\text { qualitativa; Calculou- } \\
\text { se alfa de Cronbach } \\
\text { para validação } \\
\text { interna; relacionou-se } \\
\text { a insegurança com } \\
\text { a renda familiar e } \\
\text { frequência de consumo } \\
\text { diário de alimentos } \\
\text { - Inquérito } \\
\text { populacional em } 4 \\
\text { cidades brasileiras, } \\
\text { totalizando: } 717 \\
\text { famílias urbanas e } 1.150 \\
\text { rurais }\end{array}$ & $\begin{array}{l}\text { - Alfa de Cronbach variou } \\
\text { de } 0,91 \text { a } 0,94 \text {; famílias } \\
\text { consideradas inseguras } \\
\text { possuíam menor renda, } \\
\text { menor consumo diário } \\
\text { de carne, derivados de } \\
\text { leite, frutas e verduras (p } \\
<0,01 \text { ) }\end{array}$ \\
\hline $\begin{array}{l}\text { Álvarez et } \\
\text { al. }^{12}\end{array}$ & $\begin{array}{l}\text { Escala de } \\
\text { Percepción } \\
\text { de Seguridad } \\
\text { Alimentaria } \\
\text { (EPSA) }\end{array}$ & $\begin{array}{l}\text { Colômbia, } \\
2006\end{array}$ & $\begin{array}{l}\text { Escala de Seguridad } \\
\text { Alimentaria } \\
\text { Percibida (Escala } \\
\text { venezuelana) }\end{array}$ & $\begin{array}{l}\text { - Realização de grupos } \\
\text { focais para avaliação } \\
\text { qualitativa; Aplicação } \\
\text { da escala revisada nos } \\
\text { domicílios; validação } \\
\text { interna pelo modelo } \\
\text { Rasch e análise de } \\
\text { correlação com outras } \\
\text { variáveis. } \\
\text { - Participaram } 1.624 \\
\text { domicílios com crianças } \\
\text { menores de } 10 \text { anos }\end{array}$ & $\begin{array}{l}\text { - Valores de ajuste } \\
\text { (infit) para cada um } \\
\text { dos itens no modelo } \\
\text { Rasch foram adequados; } \\
\text { a escala apresentou } \\
\text { correção inversa }(\mathrm{r}= \\
-0,650, \mathrm{p}<0,000) \text { com } \\
\text { a disponibilidade de } \\
\text { alimentos no domicílio }\end{array}$ \\
\hline
\end{tabular}


Quadro 2. continuação

\begin{tabular}{|c|c|c|c|c|c|}
\hline Referência & Escala & País, ano & $\begin{array}{c}\text { Padrão de } \\
\text { referência para } \\
\text { validação } \\
\end{array}$ & $\begin{array}{l}\text { Método de validação e } \\
\text { amostra utilizada }\end{array}$ & Principais resultados \\
\hline $\begin{array}{l}\text { Melgar- } \\
\text { Quinonez et } \\
\text { al. }^{13}\end{array}$ & $\begin{array}{l}\text { 9-item U.S. } \\
\text { Household } \\
\text { Food Security } \\
\text { Supplemental } \\
\text { Module } \\
\text { (HFSSM) version }\end{array}$ & Bolívia, 2006 & $\begin{array}{l}\text { U.S. Household } \\
\text { Food Security } \\
\text { Supplemental } \\
\text { Module (HFSSM) }\end{array}$ & $\begin{array}{l}\text { - Utilizou-se ANOVA e } \\
\text { análise de correlação } \\
\text { - } 327 \text { domicílios }\end{array}$ & $\begin{array}{l}\text { - Os domicílios seguros, } \\
\text { em comparação aos } \\
\text { inseguros, tiveram } \\
\text { maior gasto diário (p } \\
<0,01 \text { ) com os grupos } \\
\text { alimentares analisados; } \\
\text { a insegurança alimentar } \\
\text { foi inversamente } \\
\text { correlacionada ( } \mathrm{r}=-0,39 ; \\
\mathrm{p}=0,001) \text { com o gasto } \\
\text { com os alimentos }\end{array}$ \\
\hline $\begin{array}{l}\text { Hackett et } \\
\text { al. }{ }^{14}\end{array}$ & $\begin{array}{l}\text { Adult and } \\
\text { Children Food } \\
\text { Security } \\
\text { Survey }\end{array}$ & $\begin{array}{l}\text { Equador, } \\
2007\end{array}$ & $\begin{array}{l}\text { U.S. Household } \\
\text { Food Security } \\
\text { Supplemental } \\
\text { Module (HFSSM) }\end{array}$ & $\begin{array}{l}\text { - Validação interna pelo } \\
\text { Modelo Rasch; ANOVA } \\
\text { para comparação das } \\
\text { médias de aquisição } \\
\text { de alimentos, entre os } \\
\text { diferentes níveis de } \\
\text { insegurança alimentar } \\
\text {-52 domicílios }\end{array}$ & $\begin{array}{l}\text {-Os resultados indicaram } \\
\text { que quanto maior o nível } \\
\text { de insegurança alimentar, } \\
\text { menor a quantidade } \\
\text { de alimentos adquirida } \\
\text { ( } \mathrm{p}=0,003) \text {; A maioria } \\
\text { das perguntas ( }>75 \%) \\
\text { apresentaram valores de } \\
\text { infit apropriados segundo } \\
\text { o Modelo Rasch }\end{array}$ \\
\hline $\begin{array}{l}\text { Álvarez et } \\
\text { al. }^{21} \text {, } \\
\text { Pérez- } \\
\text { Escamilla et } \\
\text { al. }^{22} \text {, }\end{array}$ & $\begin{array}{l}\text { Escala Latino- } \\
\text { americana e } \\
\text { Caribenha } \\
\text { para a Medição } \\
\text { da Segurança } \\
\text { Alimentar } \\
\text { (ELCSA) }\end{array}$ & $\begin{array}{l}\text { Colômbia, } \\
\text { 2008; } \\
\text { México e } \\
\text { Uruguai, } \\
\text { 2011; }\end{array}$ & $\begin{array}{l}\text { U.S. Household } \\
\text { Food Security } \\
\text { Supplemental } \\
\text { Module (HFSSM); } \\
\text { Escala Brasileira } \\
\text { de Insegurança } \\
\text { Alimentar (EBIA); } \\
\text { Escala de Percepción } \\
\text { de Seguridad } \\
\text { Alimentaria (EPSA) }\end{array}$ & $\begin{array}{l}\text { - Para validação interna } \\
\text { da ELCSA os estudos } \\
\text { calcularam o coeficiente } \\
\text { alfa de Cronbach } \\
\text { - Colômbia: } 13.602 \\
\text { domicílios } \\
\text { - México: } 1.544 \\
\text { - Uruguai: } 700\end{array}$ & $\begin{array}{l}\text { - Valores de alfa de } \\
\text { Cronbach: } \\
\text { - Colômbia: } 0,96 \\
\text { - México: } 0,91 \\
\text { - Uruguai: 0,93 }\end{array}$ \\
\hline $\begin{array}{l}\text { González et } \\
\text { al. }{ }^{15}\end{array}$ & $\begin{array}{l}\text { Food insecurity } \\
\text { of household } \\
\text { questionnaire }\end{array}$ & $\begin{array}{l}\text { Costa Rica, } \\
2008\end{array}$ & $\begin{array}{l}\text { U.S. Household } \\
\text { Food Security } \\
\text { Supplemental } \\
\text { Module (HFSSM) }\end{array}$ & $\begin{array}{l}\text { - Calculou-se alfa } \\
\text { de Cronbach para } \\
\text { validação interna } \\
\text {-213 domicílios } \\
\end{array}$ & $\begin{array}{l}\text { - Valor de alfa de } \\
\text { Cronbach foi de } 0,89\end{array}$ \\
\hline $\begin{array}{l}\text { Melgar- } \\
\text { Quinonez et } \\
\text { al. }^{20}\end{array}$ & $\begin{array}{l}\text { Escala Mexicana } \\
\text { de Seguridad } \\
\text { Alimentaria } \\
\text { (EMSA) }\end{array}$ & México, 2008 & $\begin{array}{l}\text { Escala Latino- } \\
\text { americana e } \\
\text { Caribenha para } \\
\text { a Medição da } \\
\text { Segurança Alimentar } \\
\text { (ELCSA) }\end{array}$ & $\begin{array}{l}\text { - Modelo Rasch para } \\
\text { validação interna } \\
\text { - } 1.511 \text { domicílios }\end{array}$ & $\begin{array}{l}\text { - Valores de ajuste } \\
\text { próximos (infit) } \\
\text { apresentaram resultados } \\
\text { adequados }\end{array}$ \\
\hline $\begin{array}{l}\text { Pérez- } \\
\text { Escamilla et } \\
\text { al. }{ }^{17}\end{array}$ & $\begin{array}{l}\text { Latin American } \\
\text { and Caribbean } \\
\text { Household Food } \\
\text { Security Scale no } \\
\text { Haiti }\end{array}$ & Haiti, 2008 & $\begin{array}{l}\text { Escala Latino- } \\
\text { americana e } \\
\text { Caribenha para } \\
\text { a Medição da } \\
\text { Segurança Alimentar } \\
\text { (ELCSA) }\end{array}$ & $\begin{array}{l}\text { - Calculou-se alfa de } \\
\text { Cronbach; Modelo } \\
\text { Rasch; e análises de } \\
\text { correlação } \\
\text { - } 153 \text { domicílios }\end{array}$ & $\begin{array}{l}\text { - Valor do alfa de } \\
\text { Cronbach foi de 0,92; } \\
\text { Valores de ajuste } \\
\text { próximos (infit) } \\
\text { apresentaram resultados } \\
\text { adequados; correlação da } \\
\text { insegurança alimentar } \\
\text { com ausência de energia } \\
\text { elétrica, falta de acesso } \\
\text { à terra, e domicílios } \\
\text { chefiados por mulheres }\end{array}$ \\
\hline
\end{tabular}


Quadro 2. continuação

\begin{tabular}{|c|c|c|c|c|c|}
\hline Referência & Escala & País, ano & $\begin{array}{c}\text { Padrão de } \\
\text { referência para } \\
\text { validação }\end{array}$ & $\begin{array}{l}\text { Método de validação e } \\
\text { amostra utilizada }\end{array}$ & Principais resultados \\
\hline $\begin{array}{l}\text { Bezuneh et } \\
\text { al. }{ }^{18}\end{array}$ & $\begin{array}{l}\text { Dominican } \\
\text { Republic } \\
\text { Household Food } \\
\text { Security Survey } \\
\text { Module }\end{array}$ & $\begin{array}{l}\text { República } \\
\text { Dominicana, } \\
2008\end{array}$ & $\begin{array}{l}\text { U.S. Household } \\
\text { Food Security } \\
\text { Supplemental } \\
\text { Module (HFSSM) }\end{array}$ & $\begin{array}{l}\text { - Grupo focal para } \\
\text { avaliação qualitativa; } \\
\text { Modelo Rasch para } \\
\text { validação interna } \\
\text { - } 110 \text { domicílios }\end{array}$ & $\begin{array}{l}\text { - Valores de ajuste } \\
\text { próximos (infit) } \\
\text { apresentaram resultados } \\
\text { adequados }\end{array}$ \\
\hline Salvia etl al. ${ }^{19}$ & $\begin{array}{l}\text { Del Módulo } \\
\text { de Inseguridad } \\
\text { Alimentaria } \\
\text { Encuesta } \\
\text { de la Deuda } \\
\text { Social (EDSA) } \\
\text { Bicentenario }\end{array}$ & $\begin{array}{l}\text { Argentina, } \\
2010\end{array}$ & $\begin{array}{l}\text { Escala Latino- } \\
\text { americana e } \\
\text { Caribenha para } \\
\text { a Medição da } \\
\text { Segurança Alimentar } \\
\text { (ELCSA) }\end{array}$ & $\begin{array}{l}\text {-Calculou-se a } \\
\text { prevalência de } \\
\text { insegurança alimentar } \\
\text { e sua distribuição } \\
\text { segundo indicadores } \\
\text { socioeconômicos } \\
\text { - } 5.712 \text { domicílios } \\
\end{array}$ & $\begin{array}{l}\text { - A insegurança alimentar } \\
\text { foi mais prevalente em } \\
\text { domicílios de menor } \\
\text { renda, maior número } \\
\text { de morados e cujos } \\
\text { chefes encontravam-se } \\
\text { desempregados }(\mathrm{p}<0,05)\end{array}$ \\
\hline $\begin{array}{l}\text { Melgar- } \\
\text { Quinonez }^{20}\end{array}$ & $\begin{array}{l}\text { Escala Latino- } \\
\text { americana e } \\
\text { Caribenha } \\
\text { para a Medição } \\
\text { da Segurança } \\
\text { Alimentar } \\
\text { (ELCSA) na } \\
\text { Guatemala }\end{array}$ & $\begin{array}{l}\text { Guatemala, } \\
2010\end{array}$ & $\begin{array}{l}\text { Escala Latino- } \\
\text { americana e } \\
\text { Caribenha para } \\
\text { a Medição da } \\
\text { Segurança Alimentar } \\
\text { (ELCSA) }\end{array}$ & $\begin{array}{l}\text { - Modelo Rasch } \\
\text { para validação } \\
\text { interna; associação } \\
\text { com variáveis } \\
\text { socioeconômicas } \\
\text { (validação externa) } \\
\text { - 265.212 domicílios } \\
\text { urbanos e rurais } \\
\end{array}$ & $\begin{array}{l}\text { - Valores de ajuste (infit) } \\
\text { para cada um dos itens } \\
\text { no modelo Rasch foram } \\
\text { adequados } \\
\text { - Associou-se à pobreza, } \\
\text { condições de vida, acesso } \\
\text { a serviços públicos e posse } \\
\text { de bens }\end{array}$ \\
\hline Santos et al. ${ }^{11}$ & $\begin{array}{l}\text { Escala Brasileira } \\
\text { de Insegurança } \\
\text { Alimentar - } \\
\text { versão curta }\end{array}$ & Brasil, 2014 & $\begin{array}{l}\text { Escala Brasileira } \\
\text { de Insegurança } \\
\text { Alimentar (EBIA) - } \\
\text { versão longa }\end{array}$ & $\begin{array}{l}\text { - Calculou-se } \\
\text { sensibilidade, } \\
\text { especificidade, acurácia } \\
\text { e concordância (teste } \\
\text { de kappa) entre os dois } \\
\text { instrumentos. } \\
\text { - Os autores utilizaram } \\
\text { duas amostras para } \\
\text { testar os resultados ( } 230 \\
\text { famílias de baixa renda } \\
\text { de Pelotas-RS e } 15.575 \\
\text { mulheres da PNDS) }\end{array}$ & $\begin{array}{l}\text { - Valor de sensibilidade da } \\
\text { escala de cinco questões } \\
\text { foi de } 95,7 \% \text { e } 99,5 \% \text { na } \\
\text { amostra de Pelotas e da } \\
\text { PNDS, respectivamente. } \\
\text { O resultado do teste de } \\
\text { kappa foi de } 95 \% \text {, na } \\
\text { amostra de Pelotas, e } 99 \% \\
\text { na amostra da PNDS }\end{array}$ \\
\hline
\end{tabular}

Nota: PNDS: Pesquisa Nacional de Demografia e Saúde (2006).

A ELCSA apresenta tempo de referência de três meses para as questões da escala, semelhante à EBIA ${ }^{10}$ e Escala Mexicana de Seguridad Alimen$\operatorname{taria}^{16} ; 15$ questões sobre a percepção do indivíduo em relação ao alimento, sendo que os itens 9 a 15 são específicos para domicílios com presença de menor de 18 anos.

Com a ELCSA, países latino-americanos e caribenhos que ainda não dispunham de escalas validadas para sua população poderão utilizar essa ferramenta, assim como aqueles que validaram suas escalas utilizando padrões de referência dos EUA, poderão usufruir de um indicador mais condizente com realidade regional. A utilização de um mesmo indicador para toda a região possibilitará comparabilidade entre países, o que poderá contribuir para tomada de decisões políticas.

A experiência mundial com o uso de escalas de insegurança alimentar, em especial a ELCSA, levou a FAO, por meio do projeto "Vozes da Fome" a desenvolver a Escala de Vivência da Insegurança Alimentar (FIES - Food Insecurity Experience Scale) $)^{33}$. Tradicionalmente, a FAO 
monitora a situação de insegurança alimentar mundial através das folhas de balanço de alimentos, que são utilizadas para o cálculo da disponibilidade de energia em mais de 150 países. Essa metodologia possibilita o cálculo da prevalência de subalimentação que representa a proporção de indivíduos que não possuem suas necessidades energéticas atendidas ${ }^{34}$.

A FAO, por meio da FIES, poderá monitorar e assegurar comparabilidade global da vivência da insegurança alimentar. Esse novo indicador constitui ferramenta eficaz para avaliar as novas metas dos Objetivos de Desenvolvimento Sustentável (ODS) $)^{35}$, que se referem especificamente ao acesso a alimentos seguros e saudáveis, além de sedimentar a importância da avaliação da insegurança alimentar através de escalas de vivência que possibilitam dimensionar esse fenômeno.

\section{Considerações finais}

Sendo a segurança alimentar e nutricional um fenômeno complexo, nenhum indicador isolado é capaz de mensurar todos seus componentes. Os países da América Latina e Caribe, além das suas escalas nacionais, contam com indicador regio- nal a ELCSA, que possibilitará comparabilidade dos resultados entre os países. A análise desses resultados fornecerá informações para tomada de decisão e criação de políticas adequadas para o fortalecimento do direito humano a alimentação adequada.

A insegurança alimentar é um problema que afeta milhares de pessoas em todo mundo. Conhecer com exatidão sua localização, distribuição, determinantes e consequências é essencial para seu controle. Diante disso, destaca-se a importância da utilização de indicadores válidos, confiáveis e internacionalmente aceitos, em especial os que possibilitam dimensionar a magnitude desse fenômeno.

A experiência acumulada, desde a publicação das primeiras escalas de insegurança nos Estados Unidos, demostrou a importância desse instrumento e gerou o interesse de outros países para validação dessas escalas, em especial os da América Latina e Caribe. A exitosa experiência dos países latino-americanos e caribenhos, no desenvolvimento de escalas nacionais e uma regional, pode fortalecer o interesse dos países dessa região, e de outras partes do mundo, que ainda não possuem escalas de insegurança alimentar validadas para sua população.

\section{Colaboradores}

N Sperandio e DC Morais participaram da concepção e delineamento do estudo, redação e revisão crítica do conteúdo intelectual, aprovação da versão final do manuscrito; e SE Priore da revisão crítica do conteúdo e aprovação da versão final do manuscrito. 


\section{Referências}

1. Brasil. Lei n ${ }^{\circ} 11.346$, de 15 de setembro de 2006. Lei Orgânica de Segurança Alimentar e Nutricional. Dispõe sobre a Criação do Sistema Nacional de Segurança Alimentar e Nutricional - SISAN com vistas em assegurar o direito humano à alimentação adequada e dá outras providências. Diário Oficial da União 2006; 18 set.

2. Food and Agriculture Organization (FAO). The state of food insecurity in the world 2006: Eradicating world hunger - taking stock ten years after the World Food Summit. Rome: FAO; 2006.

3. Segall-Corrêa AM. Insegurança alimentar medida a partir da percepção das pessoas. Estud Av. 2007; 21(60):143-154.

4. Melgar-Qinonez H, Hackett M. Measuring household food security: the global experience. Rev. Nutr. 2008; 21:27-37.

5. Radimer KL, Olson CM, Campbell CC. Development of indicators to assess hunger. J Nutr 1990; 120(Supl. 11):1544-1548.

6. Wolfe WS, Frongillo EA. Building household food security measurement tools from the ground up. Food Nutr Bull 2001; 22:5-12.

7. Pérez-Escamilla R, Melgar-Quiñonez H, Nord M, Álvarez Uribe MC, Segall-Corrêa AM. Escala Latinoamericana y Caribeña de Seguridad Alimentaria (ELCSA). Perspectivas en Nutrición Humana 2007; 1:117-134.

8. Melgar-Quiñonez H, Álvarez Uribe MC, Fonseca Centeno ZY, Bermúdez O, Palma de Fulladolsa P, Fulladolsa A, Parás P, Pérez-Escamilla R. 2010. Características Psicométricas de la Escala de Seguridad Alimentaria ELCSA aplicada en Colombia, Guatemala y México. Revista de Segurança Alimentar e Nutricional 2010; 17(1):48-60.

9. Dellohain PL, Sanjur D. La adaptación y validación de una escala de seguridad alimentaria en una comunidad de Caracas, Venezuela. Arch Latinoam Nutr 2000; 50(4):2-13.

10. Pérez-Escamilla R, Segall-Corrêa AM, Kurdian Maranha L, Archanjo Sampaio MF, Marin-Leon L, Panigassi G. An adapted version of the U.S. Department of Agriculture Food Insecurity module is a valid tool for assessing household food insecurity in Campinas, Brazil. J Nutr 2004; 134(8):1923-1928.

11. Santos LP, Lindemann IL, Motta JVS, Mintem G, Bender E, Gigante DP. Proposta de versão curta da Escala Brasileira de Insegurança. Rev Saude Publica 2014; 48(5):783-789.

12. Álvarez MC, Estrada A, Montoya EC, Melgar-Quiñónez H. Validación de escala de la seguridad alimentaria doméstica en Antioquia, Colombia. Salud pública Méx 2006; 48(6):474-481.

13. Melgar-Quinonez HR, Zubieta AC, MkNelly B, Nteziyaremye A, Gerardo MF, Dunford C. Household food insecurity and food expenditure in Bolivia, Burkina Faso, and the Philippines. J Nutr 2006; 136(5):1431S-1437S.

14. Hackett M, Melgar-Quiñonez H, Zubieta AC, Hernandez K. Food Security and Household Food Supplies in Rural Ecuador. Arch Latinoam Nutr 2007; 57(1):10-17.

15. González W, Jiménez A, Madrigal G, Muñoz LM, Frongillo EA. Development and Validation of Measure of Household Food Insecurity in Urban Costa Rica Confirms Proposed Generic Questionnaire. J Nutr 2008; 138(3):587-592.
16. Consejo Nacional de la Política de Desarrollo Social (Coneval). Metodología para la medición multidimensional de la pobreza en México 2010. [acessado 2016 Mar 02]. Disponível em: http://web.coneval.gob.mx/Informes/Coordinacion/INFORMES_Y_PUBLICACIONES_PDF/Metodologia_Multidimensional_web.pdf.

17. Pérez-Escamilla R, Dessalines M, Finnigan M, Pachon H, Hromi-Fiedler A, Gupta N. Household food insecurity is associated with childhood malaria in rural Haiti. J Nutr 2009; 139(11):2132-2138.

18. Bezuneh M, Yiheyis Z, Rosario PJ, Ortiz L. Measuring Food Security in the Dominican Republic Adaptation of the U.S. Food Security Survey Module 2008. [acessado 2016 Mar 05]. Disponível em: http://naldc.nal.usda. gov/download/32857/PDF.

19. Salvia A, Tuñón I, Musante B. La Inseguridad Alimentaria en la Argentina. Hogares Urbanos. 2011. [acessado 2016 Mar 07]. Disponível em: http://www.uca.edu.ar/ uca/common/grupo68/files/Informe_Inseguridad_ Alimentaria__doc_de_trabajo_.pdf.

20. Melgar-Quinonez H. Validación de la Escala Latinoamericana y Caribeña para la medición de la Seguridad Alimentaria (ELCSA) en Guatemala. 2010. [acessado 2016 Mar 03]. Disponível em: http://coin.fao.org/coin static/cms/media/9/13155829028740/validacion_elcsa_guatemala.pdf

21. Álvarez MC, Melgar-Quiñonez H, Estrada A, Vélez OL. Validación interna y externa de la escala Latinoamericana y Caribeña para la medición de la Seguridad Alimentaria en el Hogar. Medellín: Editorial Divergráficas; 2008.

22. Pérez-Escamilla R, Parás P, Acosta MJ, Peyrou S, Nord M, Hromi-Fiedler A. Are the Latin American and Caribbean Food Security Scale (ELCSA) items comparable across countries? FASEB J 2011; 25:226-228.

23. Frongillo EA. Validation of measures of food insecurity and hunger. J Nutr 1999; 129(Supl.):506S-509S.

24. Webb P, Coates J, Frongillo EA, Rogers BL, Swindale A, Bilinsky P. Measuring household food insecurity: Why it's so important and yet so difficult to do. J Nutr 2006; 136(Supl.):1404S-1408S.

25. Kendall A, Olson CM, Frongillo Junior EA. Validation of the Radimer/Cornell measures of hunger and food insecurity. J Nutr 1995; 125(11):2793-2801.

26. Wehler CA, Scott RI, Anderson JJ. The community childhood identifi cation project: a model of domestic hunger-Demonstration project in Seattle, Washington. Journal of Nutrition Education 1992; 24(1):29S-35S.

27. Blumberg SJ, Bialostosky K, Hamilton WL, Briefel RR. The effectiveness of a short form of the Household Food Security Scale. Am J Public Health 1999; 89(8):1231-1234.

28. Instituto Brasileiro de Geografia e Estatística (IBGE). Pesquisa Nacional por Amostra de Domicílios - PNAD Segurança Alimentar: 2004. Rio de Janeiro: IBGE; 2006.

29. Instituto Brasileiro de Geografia e Estatística (IBGE) Pesquisa de Orçamento Familiar (POF):2008-2009: Antropometria e estado nutricional de crianças, adolescentes e adultos no Brasil. Rio de Janeiro: IBGE; 2010

30. Cronbach Lee J. Coefficient alpha and the internal structure of tests. Psychometrika. Springer New York 1951; 16(3):297-334. 
31. Smith EV, Conrad KM, Chang K, Piazza J. Na introduction to Rasch measurement for scale development and person assessment. J Nur Measurement 2002; 10(3):189-206.

32. Organización de las Naciones Unidas para la Alimentación y la Agricultura (FAO). Escala Latinoamericana y Caribeña de Seguridad Alimentaria (ELCSA): manual de uso y aplicaciones. Comité Científico de la ELCSA. 2012. [acessado 2016 Mar 12]. Disponível em: http:// www.fao.org/3/a-i3065s.pdf.

33. Ballard TJ, Kepple AW, Cafiero C. 2013. The food insecurity experience scale: developing a global standard for monitoring hunger worldwide. Technical Paper. Rome, FAO. [acessado 2016 Mar 12]. Disponível em: http:// www.fao.org/economic/ess/ess-fs/voices/en/.

34. Food and Agriculture Organization(FAO). The state of food insecurity in the world 2004: monitoring progress towards the world food summit and millennium development goals. Rome: FAO; 2004.

35. Programa das Nações Unidas para Desenvolvimento (PNUD). Transformando nosso mundo: a agenda 2030 para o desenvolvimento sustentável. [acessado $2016 \mathrm{Mar}$ 12]. Disponível em: http://www.pnud.org.br/Docs/ Agenda2030completo_PtBR.pdf

Artigo apresentado em 28/03/2016

Aprovado em 09/05/2016

Versão final apresentada em 11/05/2016 